\title{
GLOBULAR CLUSTER DISTANCE DETERMINATIONS
}

\author{
BRIAN CHABOYER \\ Steward Observatory, University of Arizona \\ Tucson, AZ, USA and \\ Department of Physics and Astronomy, Dartmouth College \\ Hanover, NH, USA
}

\section{Introduction}

The distance scale to globular clusters is of great interest for two principal reasons. Firstly, the best estimates for the absolute ages of globular clusters require that the distance to the clusters be known (e.g. Renzini 1991; Chaboyer 1996). These age determinations provide the best estimate for the age of the universe, but are very sensitive to the adopted globular cluster distance scale. For example, a revision in the distance scale by 0.10 mag changes the derived ages by $10 \%$. The second reason for the interest in the globular cluster distance scale is that globular clusters contain stellar populations (RR Lyrae stars, tip of the red giant branch) which are commonly used as distance indicators in astronomy. Thus, globular clusters can serve as nearby calibrators of these standard candles.

The release of the Hipparcos data set has caused a number of workers to re-examine the question of the globular cluster distance scale. Hipparcos provided high quality parallaxes for a number of nearby metal-poor stars, yielding a calibration of the absolute magnitude of metal-poor stars which could be used to derive the distance to globular clusters from main sequence fitting (Reid 1997, 1998; Gratton et al. 1997; Chaboyer et al. 1998; Grundahl et al. 1998; Pont et al. 1998). In addition, Hipparcos provided proper motions for a large number of RR Lyrae stars. These proper motions have been combined with the method of statistical parallax to estimate the absolute magnitude of RR Lyrae stars, one of the standard candles found in globular clusters (Fernley et al. 1998a).

In this chapter, the results from these studies and other recent investigations of the globular cluster distance scale will be reviewed. This is not meant to be a comprehensive review, as only results from the last few 
years are discussed in detail. The calibration of the absolute magnitude of the RR Lyrae stars is presented in $\S 2$. Astrometric distances derived from internal proper motion and radial velocity studies are discussed in $\S 3$. Section 4 contains a summary of results based upon main sequence fitting; a more complete discussion may be found in the chapter by Gratton et al. in this volume. The results of white dwarf sequence fitting are presented in $\S 5$, while the potential of other distance indicators is discussed in $\S 6$. The various results are compared in $\S 7$ which summarizes the current status of the globular cluster distance scale.

\section{RR Lyrae stars}

RR Lyrae stars are radially pulsating variable stars, which have traditionally been used as standard candles in astronomy. As RR Lyrae stars are found in many globular clusters, they are one of the primary distance indicators to globular clusters. However, RR Lyrae stars are not perfect standard candles; it has been known for many years that their absolute magnitude $\left(\mathrm{M}_{\mathrm{v}}(\mathrm{RR})\right)$ is a function of metallicity (Sandage 1981a,b). This has traditionally been parameterized as a simple linear relationship between $\mathrm{M}_{\mathrm{v}}(\mathrm{RR})$ and $[\mathrm{Fe} / \mathrm{H}]$ :

$$
\mathrm{M}_{\mathrm{v}}(\mathrm{RR})=\alpha[\mathrm{Fe} / \mathrm{H}]+\beta .
$$

There are a variety of different techniques which can be used to determine $M_{v}(R R)$, and hence calibrate RR Lyrae stars as standard candles to be used in determining the distances to globular clusters. Some of these methods are best for ascertaining the zero-point of the $\mathrm{M}_{\mathrm{v}}(\mathrm{RR})$-metallicity relation $(\beta)$, while others are best in determining the variation of $M_{v}(R R)$ with metallicity $(\alpha)$. The determination of each of these quantities will be discussed in turn.

\subsection{VARIATION OF $\mathrm{M}_{\mathrm{v}}(\mathrm{RR})$ WITH METALLICITY}

Sandage (1981a,b) first derived the coefficients in equation (1) empirically, and estimated a "steep" slope $\alpha$ of 0.35 . From a theoretical calibration based on synthetic horizontal branch (HB) population models, Lee et al. $(1990,1994)$ derived a "shallower" $\alpha$ in the range 0.17-0.19. It is important to note that theoretical models predict that a simple linear relationship between $\mathrm{M}_{\mathrm{v}}(\mathrm{RR})$ and $[\mathrm{Fe} / \mathrm{H}]$ does not exist. Theory predicts that stars of the same metallicity evolve through the RR Lyrae instability strip at different luminosities depending on whether they originate on the red or blue side of the instability strip. Thus $\mathrm{M}_{\mathrm{v}}(\mathrm{RR})$ depends on $\mathrm{HB}$ morphology. At a given metallicity, RR Lyrae variables are more luminous in clusters with 
blue HB morphology types than in red HB morphology type (Lee 1991). This difficulty suggests that the standard $M_{v}(R R)$ calibration should not be applied to globular clusters with extremely blue HB morphologies (e.g. see Fig. 1 in Caputo 1997). Furthermore, theoretical HB models do not predict a simple linear relationship between $\mathrm{M}_{\mathrm{V}}(\mathrm{RR})$ and $[\mathrm{Fe} / \mathrm{H}]$ even among globular clusters with similar HB types (Caputo 1997; Caloi et al. 1997). For example, with $[\alpha / \mathrm{Fe}]=+0.4$, Caputo (1997) predicts $\alpha=0.19$ for $[\mathrm{Fe} / \mathrm{H}]<-1.6$ and $\alpha=0.32$ for $[\mathrm{Fe} / \mathrm{H}]>-1.6$. The large differences between these two slopes, suggest that the traditional assumption of a simple linear relationship between $\mathrm{M}_{\mathrm{v}}(\mathrm{RR})$ and $[\mathrm{Fe} / \mathrm{H}]$ is not valid. This is certainly true in detail, but a global fit over the range of metallicities typically found in globular clusters with RR Lyrae stars $(-2.2 \leq[\mathrm{Fe} / \mathrm{H}] \leq-1.0)$ finds $\alpha=0.25$, with a maximum deviation of $0.02 \mathrm{mag}$ in $\mathrm{M}_{\mathrm{v}}(\mathrm{RR})$. Even a fit over a very broad metallicity range $(-2.2 \leq[\mathrm{Fe} / \mathrm{H}] \leq 0.0)$ leads to maximum deviations of only 0.04 mag in $\mathrm{M}_{\mathrm{v}}(\mathrm{RR})$ between a simple linear fit and the relationship derived directly from the models. Given the small residuals between the linear fit and the $\mathrm{M}_{\mathrm{v}}(\mathrm{RR})$ values predicted by theoretical models, it is justified to assume a linear relation between $M_{v}(R R)$ and $[\mathrm{Fe} / \mathrm{H}]$ for distance determinations.

The semi-empirical Baade-Wesselink method supports a shallow slope, $\alpha=0.20 \pm 0.04$ (Fernley et al. 1998b). Note that the Baade-Wesselink results include many high metallicity points $([\mathrm{Fe} / \mathrm{H}] \geq 0.5)$ and the theoretical models would predict a bias to a higher slope. The shallow slope is also supported by HST observations of globular cluster HBs in M31, where $\alpha=0.13 \pm 0.07$ (Fusi Pecci et al.1996). The clusters span a range in metallicity $(-1.8 \leq[\mathrm{Fe} / \mathrm{H}] \leq-0.4)$ which is unlikely to introduce a significant bias to the derived value of $\alpha$. Using the relation between Fourier decomposition and luminosity for RRab stars in globular clusters Kovács \& Jurcsik (1996) determined that $\alpha$ is less than 0.20. Overall, the observations and theoretical models appear to favor somewhat shallow slopes $(\alpha \leq 0.26)$. In the rest of this review a value of $\alpha=0.23 \pm 0.04$ is adopted. The 1- $\sigma$ range of this value $(0.19-0.27)$ encompasses the majority of recent determinations of $\alpha$.

\subsection{ZERO-POINT OF $\mathrm{M}_{\mathrm{v}}(\mathrm{RR})$ WITH METALLICITY}

\subsubsection{Statistical Parallax}

A traditional method used to determine the absolute magnitude of RR Lyrae stars is statistical parallax (see Layden this volume). This method determines the absolute magnitude of RR Lyrae stars in the field. Hipparcos obtained a large number of proper motions which can be used in the statistical parallax solution. Current statistical parallax solutions find 
$\mathrm{M}_{\mathrm{v}}(\mathrm{RR})=0.77 \pm 0.13 \mathrm{mag}$ at $<[\mathrm{Fe} / \mathrm{H}]>=-1.60$ (Gould \& Popowski 1998; also Layden, this volume). Combining this with the estimate for the slope given in the previous section yields

$$
\mathrm{M}_{\mathrm{v}}(\mathrm{RR})=(0.23 \pm 0.04)([\mathrm{Fe} / \mathrm{H}]+1.6)+(0.77 \pm 0.13) .
$$

\subsubsection{Calibration via the $L M C$}

Given a distance estimate to the LMC, the observed magnitude of RR Lyrae stars in the LMC can be used to calibrate $\mathrm{M}_{\mathrm{v}}(\mathrm{RR})$ (e.g. Walker 1992). Walker (this volume) summarizes current distance estimates to the LMC, and concludes that the distance modulus to the LMC is $18.55 \pm 0.10$ mag. Walker (1992) determined the mean magnitude of a large number of RR Lyrae stars in several clusters in the LMC. Combining this data with the above distance modulus to the $\mathrm{LMC}$ yields $\mathrm{M}_{\mathrm{v}}(\mathrm{RR})=0.39 \pm 0.10 \mathrm{mag}$ at $\langle[\mathrm{Fe} / \mathrm{H}]\rangle=-1.90$. With $\alpha=0.23 \pm 0.04$ this yields

$$
\mathrm{M}_{\mathrm{v}}(\mathrm{RR})=(0.23 \pm 0.04)([\mathrm{Fe} / \mathrm{H}]+1.6)+(0.46 \pm 0.11)
$$

(allowing for an error of 0.15 dex in the mean LMC $[\mathrm{Fe} / \mathrm{H}]$ ). A comparison between equations (2) and (3) indicates that these two methods for determining the zero-point of the $\mathrm{M}_{\mathrm{v}}(\mathrm{RR})-[\mathrm{Fe} / \mathrm{H}]$ relation differ by $1.8 \sigma$.

\subsubsection{Theoretical HB Models}

Theoretical stellar evolution models may be used to derive the absolute magnitude of the zero-age horizontal branch (ZAHB). It is important to note that the results of these calculations depend sensitively on the assumed helium abundance used in the calculations, along with the physics used in the construction of the stellar models. A change in the assumed main sequence helium abundance by $4 \%$ (from $Y=0.23$ to $Y=0.24$ for example) leads to a change in the predicted HB luminosity of approximately 0.05 mag. Cassisi et al. (1998) show that improvements in the physics used in the theoretical models over the last 10 years has lead to an increase in the predicted ZAHB luminosity by about $0.15 \mathrm{mag}$. For this reason, only globular cluster distance determinations based upon the latest input physics will be considered in this subsection.

A number of authors have used theoretical ZAHB models to derive the distance to specific globular clusters. Brocato et al. (1997) constructed ZAHB models for M68, and compared these to the observations obtained by Walker (1994). The existence of a blue tail on the M68 HB allowed Brocato et al. (1997) to derive the distance and reddening to M68 simultaneously. They obtained $(\mathrm{m}-\mathrm{M})_{\mathrm{V}}=15.25 \mathrm{mag}$ and $\mathrm{E}(\mathrm{B}-\mathrm{V})=0.05$ using their most recent models. Walker (1994) obtained a mean apparent magnitude 
of $V=15.67 \pm 0.04 \mathrm{mag}$ for the RR Lyrae stars in M68. Estimates of the metallicity of $\mathrm{M} 68$ vary from $[\mathrm{Fe} / \mathrm{H}]=-2.17$ (Minniti et al. 1993) to $[\mathrm{Fe} / \mathrm{H}]=-1.99$ (Carretta \& Gratton 1997). Taking the average of these two metallicity estimates, the distance modulus derived by Brocato et al. (1997) implies $\mathrm{M}_{\mathrm{v}}(\mathrm{RR})=0.42 \mathrm{mag}$ at $[\mathrm{Fe} / \mathrm{H}]=-2.08$.

Salaris et al. (1997) performed a fit to M68 using their ZAHB models and isochrones. The distance modulus and reddening were determined by shifting the ZAHB models and isochrones in order to match the observed mainsequence ridge line and the ZAHB level in the RR Lyrae region. Salaris et al. (1997) obtained $(\mathrm{m}-\mathrm{M})_{\mathrm{V}}=15.26 \mathrm{mag}$ and $\mathrm{E}(\mathrm{B}-\mathrm{V})=0.06$. This implies $\mathrm{M}_{\mathrm{v}}(\mathrm{RR})=0.41 \mathrm{mag}$, which is very similar to the result obtained by Brocato et al. (1997).

Finally, Caloi et al. (1997) have used their ZAHB models to determine the distance to three globular clusters. Their models differ from other workers in that they do not use mixing length theory, but adopt the Canuto \& Mazzitelli (1991) convection treatment. Using the same M68 data, Caloi et al. (1997) determined a distance modulus of $(\mathrm{m}-\mathrm{M})_{\mathrm{V}}=15.37 \mathrm{mag}$ (assuming $A_{V}=3.2 \mathrm{E}(\mathrm{B}-\mathrm{V})$ ). This is 0.11 mag larger than the value found by Brocato et al. (1997) and Salaris et al. (1997). However, the work of Caloi et al. (1997) ignored the fact that the $\alpha$ capture elements are enhanced over their solar ratio in metal poor stars (e.g. Nissen et al. 1994). Caloi et al. (1997) used their $Z=0.0001$ models to compare to M68, while Salaris et al. (1997) and Brocato et al. (1997) take into account $\alpha$ element enhancement by using their $Z=0.0002$ models. The results of Caloi et al. (1997) may be corrected to include $\alpha$ element enhancement with the aid of their Table 2. Performing such a correction leads to $(\mathrm{m}-\mathrm{M})_{\mathrm{V}}=15.28$ mag, implying $\mathrm{M}_{\mathrm{V}}(\mathrm{RR})=0.39 \mathrm{mag}$ in good agreement with Brocato et al. (1997) and Salaris et al. (1997). Averaging these three determinations for the distance to $\mathrm{M} 68$ yields $\mathrm{M}_{\mathrm{v}}(\mathrm{RR})=0.41 \mathrm{mag}$ at $[\mathrm{Fe} / \mathrm{H}]=-2.08$ based upon theoretical ZAHB models.

Caloi et al. (1997) also derived the distance to M5. Once again, correcting their published value for the effects of $\alpha$ element enhancement leads to a distance modulus of $(\mathrm{m}-\mathrm{M})_{\mathrm{V}}=14.51 \mathrm{mag}$ for M5. M5 has a metallicity of $[\mathrm{Fe} / \mathrm{H}]=-1.17$ from high dispersion spectroscopic analysis (Sneden et al.1992) and mean RR Lyrae apparent magnitude of $V=15.05 \pm 0.06$ mag (Reid 1996). Thus, the theoretical ZAHB models of Caloi et al. (1997) imply $\mathrm{M}_{\mathrm{v}}(\mathrm{RR})=0.56 \mathrm{mag}$ at $[\mathrm{Fe} / \mathrm{H}]=-1.17$. This may be combined with the M68 calibration above to yield a calibration of $\mathrm{M}_{\mathrm{v}}(\mathrm{RR})$ based upon theoretical ZAHB models from three different groups:

$$
\mathrm{M}_{\mathrm{v}}(\mathrm{RR})=(0.23 \pm 0.04)([\mathrm{Fe} / \mathrm{H}]+1.6)+(0.49 \pm 0.10) .
$$

The error in the zero-point has been estimated from a consideration of the 
uncertainties associated with the theoretical HB models, discussed in the beginning of this section.

\section{Astrometric Distances}

A comparison of the proper motion and radial velocity dispersions within a cluster allows for a direct determination of GC distances, independent of reddening (Cudworth 1979). Although this method requires that a dynamical model of a cluster be constructed, it is the only method considered here which directly measures the distance to a GC without the use of a 'standard' candle. The chief disadvantage of this technique is its relatively low precision. This problem is avoided by averaging together the astrometric distances to a number of different GCs. Rees (1996) presents new astrometric distances to eight GCs, along with two previous determinations. As pointed out by Rees, there are possibly large systematic errors in the dynamical modeling of M15, NGC 6397 and 47 Tuc. As such, these clusters will be excluded from our analysis. Rees (private communication) has performed a new reduction of the M2 proper motions, yielding a total of seven clusters whose distances have been estimated astrometrically.

Table 1 tabulates the astrometric distances from Rees (1996) along with the new distance determination to M2. Unless otherwise noted, the numbers are those given by Rees (1996). For the $[\mathrm{Fe} / \mathrm{H}]$ values, preference has been given to the high dispersion results of Kraft, Sneden and collaborators. Table 1 also includes the HB type of the clusters taken from Harris (1996). This is defined to be $(B-R) /(B+V+R)$, where $B, V$ and $R$ are the numbers of blue, variable and red HB stars. Taking the weighted average of the $\mathrm{M}_{\mathrm{v}}(\mathrm{RR})$ values listed in Table 1 results in $\mathrm{M}_{\mathrm{v}}(\mathrm{RR})=0.60 \pm 0.10 \mathrm{mag}$ at $\langle[\mathrm{Fe} / \mathrm{H}]\rangle=-1.60$, where the average $[\mathrm{Fe} / \mathrm{H}]$ value has been calculated using the same weights as in the $\mathrm{M}_{\mathrm{v}}(\mathrm{RR})$ average. This implies

$$
\mathrm{M}_{\mathrm{v}}(\mathrm{RR})=(0.23 \pm 0.04)([\mathrm{Fe} / \mathrm{H}]+1.6)+(0.60 \pm 0.10)
$$

using the $\mathrm{M}_{\mathrm{v}}(\mathrm{RR})-[\mathrm{Fe} / \mathrm{H}]$ slope adopted in $\S 2.1$.

The four most metal-poor clusters all have very blue HB types. For these clusters, theoretical models suggest that the RR Lyrae stars will be more luminous than for stars with redder HB types. An average of the blue HB clusters finds $\mathrm{M}_{\mathrm{v}}(\mathrm{RR})=0.59 \pm 0.12$ at $<[\mathrm{Fe} / \mathrm{H}]>=-1.71$, while the three clusters with redder $\mathrm{HB}$ types yield $\mathrm{M}_{\mathrm{v}}(\mathrm{RR})=0.64 \pm 0.19$ at

$<[\mathrm{Fe} / \mathrm{H}]>=-1.31$. Translating these two estimates to $[\mathrm{Fe} / \mathrm{H}]=-1.60$ (using $\alpha=0.23 \pm 0.04$ ) yields $\mathrm{M}_{\mathrm{v}}(\mathrm{RR})=0.62 \pm 0.12$ for the blue $\mathrm{HB}$ clusters and $\mathrm{M}_{\mathrm{v}}(\mathrm{RR})=0.57 \pm 0.19$ for the other clusters. There does not appear to be a significant difference between the two $M_{v}(R R)$ calibrations, and so the averaging used to derive equation (5) appears to be valid. 
TABLE 1. Astrometric Distances

\begin{tabular}{lccccc}
\hline Cluster & {$[\mathrm{Fe} / \mathrm{H}]$} & HB Type & $(\mathrm{m}-\mathrm{M})_{\mathrm{O}}$ & $\mathrm{V}(\mathrm{HB})$ & $\mathrm{M}_{\mathrm{V}}(\mathrm{HB})$ \\
\hline $\mathrm{M} 5^{a}$ & -1.17 & +0.31 & 14.44 & 15.05 & $0.51 \pm 0.41$ \\
$\mathrm{M}^{b}$ & -1.33 & -0.06 & 11.18 & 13.37 & $0.67 \pm 0.23$ \\
$\mathrm{M}^{c}$ & -1.47 & +0.08 & 14.91 & 15.63 & $0.69 \pm 0.59$ \\
$\mathrm{M}^{d} 3^{d}$ & -1.58 & +0.97 & 14.06 & 14.83 & $0.71 \pm 0.23$ \\
$\mathrm{M}^{e}$ & -1.62 & +0.96 & 15.26 & 16.05 & $0.63 \pm 0.25$ \\
$\mathrm{M}^{b} 2^{b}$ & -1.75 & +0.91 & 12.17 & 14.10 & $0.58 \pm 0.19$ \\
$\mathrm{M}^{f}$ & -2.25 & +0.91 & 14.76 & 15.13 & $0.31 \pm 0.32$ \\
\hline
\end{tabular}

${ }^{a}[\mathrm{Fe} / \mathrm{H}]$ from Sneden et al. (1992).

${ }^{b}[\mathrm{Fe} / \mathrm{H}]$ from Zinn \& West (1984).

${ }^{c} \mathrm{~V}(\mathrm{HB})$ from Buonanno et al. (1994). Reddening from Zinn (1985). [Fe/H] from Kraft et al. (1992).

${ }^{d} \mathrm{~V}(\mathrm{HB})$ from Buonanno et al. (1989). [Fe/H] from Kraft et al. (1997)

${ }^{e} \mathrm{~V}(\mathrm{HB})$ from Harris (1996). [Fe/H] from Zinn \& West (1984)

${ }^{f}[\mathrm{Fe} / \mathrm{H}]$ from Sneden et al. (1991).

\section{Main Sequence Fitting}

Hipparcos provided high quality parallaxes for a number of metal-poor field stars. This has prompted a number of authors to determine new distances to globular clusters using main sequence fitting. Main sequence fitting is discussed in detail in the chapter by Gratton et al. in this book. The results of the published investigations are summarized in Table 2. The typical distance modulus errors quoted by the various authors is \pm 0.10 mag. The authors took quite different approaches in dealing with issues such as sample selection, reddening, biases, etc. In general, the distance moduli derived by various authors for a given globular cluster are in good agreement. For example, the various distance modulus estimates to M13 agree to within \pm 0.03 mag. The Grundahl et al. (1998) distance estimate to M13 is particularly noteworthy as they utilized Strömgren photometry, while the other authors used B,V photometry. Of course, all of these work utilize the same basic assumption, that the nearby metal-poor stars have identical properties to their metal-poor counterparts in globular clusters.

Some of the globular clusters listed in Table 2 have very good RR Lyrae mean magnitudes, and so the main sequence fitting distances may be compared amongst each other, and to other methods using $\mathrm{M}_{\mathrm{v}}(\mathrm{RR})$ (equation 1). For example M92 has a mean RR Lyrae magnitude of $V=15.10 \pm 0.03$ mag (Carney et al. 1992). Averaging the distance moduli obtained by Gratton et al. (1997) and Pont et al. (1998) yields $\mathrm{M}_{\mathrm{v}}(\mathrm{RR})=0.33 \pm 0.10 \mathrm{mag}$ 
TABLE 2. Main Sequence Fitting Distances

\begin{tabular}{|c|c|c|c|c|}
\hline Cluster & $(\mathrm{m}-\mathrm{M})_{\mathrm{O}}$ & $\mathrm{E}(\mathrm{B}-\mathrm{V})$ & $(\mathrm{m}-\mathrm{M})_{\mathrm{V}}$ & Reference \\
\hline \multirow[t]{2}{*}{47 Tuc NGC 104} & 13.56 & 0.04 & 13.69 & 1 \\
\hline & 13.44 & 0.055 & 13.62 & 2 \\
\hline \multirow[t]{2}{*}{ NGC 288} & 15.00 & 0.01 & 15.03 & 1 \\
\hline & 14.83 & 0.033 & 14.94 & 2 \\
\hline NGC 362 & 14.86 & 0.056 & 15.04 & 2 \\
\hline M68 NGC 4590 & 15.18 & 0.040 & 15.31 & 2 \\
\hline \multirow[t]{3}{*}{ M5 NGC 5904} & 14.52 & 0.02 & 14.58 & 1 \\
\hline & 14.41 & 0.03 & 14.51 & 3 \\
\hline & 14.49 & 0.035 & 14.60 & 2 \\
\hline \multirow[t]{4}{*}{ M13 NGC 6205} & 14.38 & 0.021 & 14.45 & 4 \\
\hline & 14.45 & 0.02 & 14.51 & 1 \\
\hline & 14.41 & 0.02 & 14.47 & 3 \\
\hline & 14.39 & 0.020 & 14.45 & 2 \\
\hline \multirow[t]{2}{*}{ M92 NGC 6341} & 14.72 & 0.025 & 14.80 & 2 \\
\hline & 14.68 & 0.02 & 14.74 & $5^{a}$ \\
\hline NGC 6397 & 12.24 & 0.19 & 12.85 & 1 \\
\hline \multirow[t]{3}{*}{ NGC 6752} & 13.16 & 0.04 & 13.29 & 1 \\
\hline & 13.20 & 0.04 & 13.33 & 3 \\
\hline & 13.21 & 0.035 & 13.32 & 2 \\
\hline M71 NGC 6838 & 13.19 & 0.28 & 14.09 & 1 \\
\hline M30 NGC 7099 & 14.82 & 0.039 & 14.94 & 2 \\
\hline
\end{tabular}

REFERENCES. - (1) Reid 1998; (2) Gratton et al. (1997); (3) Chaboyer et al. (1998); (4) Grundahl et al. (1998); (5) Pont et al. (1998).

${ }^{a}$ This is the distance modulus derived by Pont et al. when they do not include the known binaries in their fit.

(at $[\mathrm{Fe} / \mathrm{H}]=-2.25$ from Sneden et al. 1991). The Gratton et al. (1997) distance modulus to $\mathrm{M} 68$ yields $\mathrm{M}_{\mathrm{v}}(\mathrm{RR})=0.36 \pm 0.10 \mathrm{mag}($ at $[\mathrm{Fe} / \mathrm{H}]=-2.08$ using the data for M68 given in $\S 2.2 .3)$. These two estimates for $M_{v}(R R)$ can be directly compared at an intermediate metallicity $([\mathrm{Fe} / \mathrm{H}]=-2.16)$ using equation 1 which yields $\mathrm{M}_{\mathrm{v}}(\mathrm{RR})=0.35 \pm 0.10 \mathrm{mag}$ for $\mathrm{M} 92$ and $\mathrm{M}_{\mathrm{v}}(\mathrm{RR})=0.34 \pm 0.10 \mathrm{mag}$ for M68. Note that M92 has a blue HB (HBtype of +0.91 ), while the M68 has a much redder HB (HB-type of 0.17 ). This comparison indicates, that for these two clusters the HB type does not have a significant effect on $\mathrm{M}_{\mathrm{v}}(\mathrm{RR})$.

Good RR Lyrae photometry also exists for M5 (see references in §2.2.3). 
Averaging together the three main sequence fitting results for M5 presented in Table 2 results in $(\mathrm{m}-\mathrm{M})_{\mathrm{V}}=14.56 \pm 0.10 \mathrm{mag}$ and $\mathrm{M}_{\mathrm{v}}(\mathrm{RR})=0.49 \pm$ $0.10 \mathrm{mag}$ at $[\mathrm{Fe} / \mathrm{H}]=-1.17$. Taking the mean determination of $\mathrm{M}_{\mathrm{v}}(\mathrm{RR})$ for M92, M68 and M5 from main sequence fitting (and using $\alpha=0.23 \pm 0.04$ in equation 1) yields

$$
\mathrm{M}_{\mathrm{v}}(\mathrm{RR})=(0.23 \pm 0.04)([\mathrm{Fe} / \mathrm{H}]+1.6)+(0.45 \pm 0.10) .
$$

\section{White Dwarf Fitting}

Renzini et al. (1996) have utilized deep HST WFPC2 observations of NGC 6752 to obtain accurate photometry of the cluster white dwarfs. In addition, they obtained similar photometry of nearby white dwarfs which appear to have similar masses to the cluster white dwarfs. Using the parallaxes of the nearby white dwarfs to determine their absolute magnitude, they determined the distance to NGC 6752 using a procedure similar to main sequence fitting. The key assumption in this method is that the masses of the local white dwarfs are similar to the masses of the white dwarfs in NGC 6752. The derived distance modulus is $(\mathrm{m}-\mathrm{M})_{\mathrm{V}}=13.18 \pm 0.10 \mathrm{mag}$ assuming $\mathrm{E}(\mathrm{B}-\mathrm{V})=0.04$. This reddening estimate is from Zinn (1985), and is identical to those found by Burnstein \& Heiles (1982) and Carney (1979).

The average distance modulus for NGC 6752 from main sequence fitting is $(\mathrm{m}-\mathrm{M})_{\mathrm{V}}=13.31 \pm 0.10 \mathrm{mag}$ (Table 2), leading to a difference of 0.13 mag between the main sequence and white dwarf fitting distance estimates

to NGC 6752. This cluster has a very blue HB, and so determination of its HB magnitude at the position of the RR Lyrae instability strip is very difficult. In order to compare white dwarf fitting to the other distance determination techniques, equation (6) can be combined with the difference between the white dwarf and main sequence fitting distances to NGC 6752 to yield

$$
\mathrm{M}_{\mathrm{v}}(\mathrm{RR})=(0.23 \pm 0.04)([\mathrm{Fe} / \mathrm{H}]+1.6)+(0.58 \pm 0.10) .
$$

\section{Other Distance Indicators}

There are a variety of other methods which have been used to obtain distances to globular clusters. Jimenez \& Padoan (1998) have compared theoretical luminosity functions to observed luminosity functions of M5 and M55. For M5, they determined $(\mathrm{m}-\mathrm{M})_{\mathrm{V}}=14.55 \pm 0.10$ mag. This can be compared to the average distance modulus derived from main sequence fitting of $(\mathrm{m}-\mathrm{M})_{\mathrm{V}}=14.56 \pm 0.10 \mathrm{mag}$ (Table 2). 
Kovács and Walker (1998) have presented a detailed analysis of doublemode RR Lyrae stars in M15, M68 and IC 4499. This analysis is based upon linear pulsation models and is free of systematic effects due to ambiguities in the various zero-points (bolometric corrections, magnitudes, etc). The derived absolute magnitudes are $0.2-0.3$ mag brighter than corresponding Baade-Wesselink values which are tied to the statistical parallax zero-point.

Simon \& Clement (1993) used hydrodynamic pulsation models to show that physical properties (such as absolute magnitude) of RRc stars could be derived from their pulsation period and Fourier phase parameters. Kaluzny et al. (1998) present $\mathrm{M}_{\mathrm{v}}(\mathrm{RR})$ for seven globular clusters based upon this method. For example, for M68, they find $M_{v}(R R)=0.38$ mag, which compares to $\mathrm{M}_{\mathrm{v}}(\mathrm{RR})=0.41$ mag from theoretical HB models ( $\left.\$ 2.2 .3\right)$ and $\mathrm{M}_{\mathrm{v}}(\mathrm{RR})=0.36 \mathrm{mag}$ from main sequence fitting ( $\S 5$ ). For M5, Kaluzny et al. (1998) tabulate $\mathrm{M}_{\mathrm{v}}(\mathrm{RR})=0.61 \mathrm{mag}$ which agrees well with the theoretical HB models $\left(\mathrm{M}_{\mathrm{v}}(\mathrm{RR})=0.56 \mathrm{mag}\right)$ and is somewhat fainter than that derived from main sequence fitting $\left(\mathrm{M}_{\mathrm{v}}(\mathrm{RR})=0.49 \mathrm{mag}\right)$.

The discovery of a detached eclipsing binary system within a globular cluster would allow for a near direct distance determination to the globular cluster (Paczyński 1997). If the binary is well detached and uncomplicated, accurate photometry and radial velocities can be combined with a surface brightness-color relation to obtain the distance to the globular cluster. A number of authors have searched for such binaries in globular clusters (e.g. Yan \& Mateo 1994, McVean et al. 1997, Kaluzny et al. 1998). McVean et al. (1997) have identified one eclipsing binary system in the globular cluster M71 which appears to be a detached or semi-detached system, with the detached model being more likely. Detached eclipsing binary systems have great potential as distance indicators to globular clusters which will (hopefully) be realized in the next few years.

\section{Summary}

The release of the Hipparcos data set has led a number of authors to study the distance scale to globular clusters. The Hipparcos data set of high quality parallaxes for a number of nearby metal-poor stars has renewed interest in the use of main sequence fitting to determine distances to globular clusters. In addition, the Hipparcos data on proper motions of field RR Lyrae stars has been used to determine a new calibration of the absolute magnitude of the RR Lyrae stars (via the statistical parallax method) which can be used to determine the distances to globular clusters. Over the last few years, a variety of other methods have been used to derive distances to globular clusters. Given that many globular clusters contain RR Lyrae stars, these distance determinations can be compared via their calibration of the 
absolute magnitude of the RR Lyrae stars. This calibration is presented in equations (2) - (7) and summarized in Table 3.

TABLE 3. $\quad \mathrm{M}_{\mathrm{v}}(\mathrm{RR})$ at $[\mathrm{Fe} / \mathrm{H}]=-1.6$

\begin{tabular}{lc}
\hline \multicolumn{1}{c}{ Method } & $\mathrm{M}_{\mathrm{v}}(\mathrm{RR})$ \\
\hline Statistical Parallax & $0.77 \pm 0.13$ \\
Astrometric Distances & $0.60 \pm 0.10$ \\
White Dwarf Fitting & $0.58 \pm 0.10$ \\
Theoretical HB models & $0.49 \pm 0.10$ \\
LMC & $0.46 \pm 0.11$ \\
Main Sequence Fitting & $0.45 \pm 0.10$ \\
\hline
\end{tabular}

The various calibrations fall into three groups. Main sequence fitting using Hipparcos parallaxes, theoretical HB models and the RR Lyrae in the LMC all favor a bright calibration, implying a 'long' globular cluster distance scale. White dwarf fitting and the astrometric distances yield a somewhat fainter RR Lyrae calibration, while the statistical parallax solution yields faint RR Lyrae stars implying a 'short' distance scale to globular clusters. The various secondary distance indicators discussed in $\S 6$ all favor the long distance scale. It is interesting to note that Hipparcos provides support for both the long (from main sequence fitting) and short distance scales (from statistical parallax).

A straight average of all six calibrations presented in Table 3 yields $\mathrm{M}_{\mathrm{v}}(\mathrm{RR})=0.56 \mathrm{mag}$ with a standard deviation of 0.12 mag. If the statistical parallax solution is removed from the average, then $M_{v}(R R)=0.52$ mag with a standard deviation of 0.07 mag. At the present time, their is no reason to doubt the validity of the statistical parallax solution. A number of authors, using a variety of data sources have all reached similar conclusions (see Layden, this volume). A possible explanation for the different result obtained using statistical parallax compared to the other methods is that it is the only method which calibrates the field RR Lyrae population (as opposed to the RR Lyrae stars in a globular cluster). Perhaps there is a systematic difference between the field and globular cluster RR Lyrae populations. However, a study of the pulsation properties of RR Lyrae variables in the field and in globular clusters found essentially indistinguishable period-temperature distributions for the two populations, suggesting that there is no significant difference in luminosity between them (Catelan 1998). For the above reasons, it appears prudent at this time to include the statistical parallax solution in the average. This leads to a best estimate of the $\mathrm{M}_{\mathrm{v}}(\mathrm{RR})$ calibration which can be used to set the globular cluster distance 
scale of

$$
\mathrm{M}_{\mathrm{v}}(\mathrm{RR})=(0.23 \pm 0.04)([\mathrm{Fe} / \mathrm{H}]+1.6)+(0.56 \pm 0.12),
$$

where the standard deviation among the six independent distance techniques has been used as the error in the zero-point. This is 0.1 mag fainter than that obtained from main sequence fitting, but is 0.2 mag brighter than the statistical parallax solution. Equation (8) may be compared to my best estimate for the the calibration of the RR Lyrae distance scale prior to the release of the Hipparcos data which implied $\mathrm{M}_{\mathrm{v}}(\mathrm{RR})=0.66 \pm 0.10 \mathrm{mag}$ at $[\mathrm{Fe} / \mathrm{H}]=-1.6$ (Chaboyer et al. 1996).

The impact of this distance scale on the mean age of the oldest globular clusters can be evaluated using the formulae presented by Chaboyer et al. (1998) in the caption to their Figure 3. From this, equation (8) implies a mean age of the oldest globular clusters of $13 \pm 2$ Gyr. The dominant uncertainty in this age estimate is the uncertainty in the distance scale to the globular clusters. In order to reduce the uncertainty in the absolute ages of the globular clusters, the differences between the 'long' distance scale (based upon main sequence fitting, theoretical HB models and the RR Lyrae in the LMC) and the 'short' distance scale (based upon the statistical parallax method) must be reconciled.

\section{References}

Brocato, E., Castellani, V. \& Piersimoni, A. 1997, ApJ, 491, 789

Buonanno, R., Corsi, C. E., Cacciari, C., Ferraro, F.R. \& Fusi Pecci, F. 1994, A\&A, 290, 69

Buonanno, R., Corsi, C. E. \& Fusi Pecci, F. 1989, A\&A, 216, 80

Burnstein, D. \& Heiles, C. 1982, AJ, 87, 1165

Caloi, V., D’Antona, F. \& Mazzitelli, I. 1997, A\&A, 320, 823

Canuto V.M. \& Mazzitelli, I. 1991, ApJ, 370, 295

Caputo, F. 1997, MNRAS, 284, 994

Carney, B.W. 1979, AJ, 84, 515

Carney, B.W., Storm, J., Trammell, S.R. \& Jones, R.V. 1992, PASP, 104, 44

Carretta, E. \& Gratton, R.G. 1997, A\&AS, 121, 95

Cassisi, S., Castellani, V, Degl'Innocenti, S. \& Weiss, A. 1998, A\&AS, 129, 267

Catelan, M. 1998, ApJ, 495, L81

Chaboyer, B. 1996, Nuclear Physics B Proceedings Supplement, 51B, 10

Chaboyer, B., Demarque, P., Kernan, P.J. \& Krauss, L.M. 1996, Science, 271,957

Chaboyer, B., Demarque, P., Kernan, P.J. \& Krauss, L.M. 1998, ApJ, 494, 96 
Cudworth, K.M. 1979, AJ, 84, 1212

Fernley, J., Barnes, T.G., Skillen, I., Hawley, S.L., Hanley, C.J., Evans, D.W., Solano, E. \& Garrido, R. 1998a, A\&A, 330, 515

Fernley, J., Carney, B.W. Skillen, I. Cacciari, C. \& Janes, K. 1998b, MNRAS, 293, L61

Fusi Pecci, F., Buonanno, R., Cacciari, C., Corsi, C. E., Djorgovski, S. G., Federici, L., Ferraro, F. R., Parmeggiani, G., \& Rich, R. M. 1996, AJ, 112,1461

Gould, A. \& Popowski, P. 1998, ApJ, in press

Gratton, R.G., Fusi Pecci, F., Carretta, E., Clementini, G., Corsi, C.E. \& Lattanzi, M. 1997, ApJ, 491, 749

Grundahl, F., VandenBerg, D.A. \& Andersen, M.I. 1998, ApJ, 500, L179

Harris, W.E. 1996, AJ, 112, 1487

Jimenez, R. \& Padoan, P. 1998, ApJ, 498, 704

Kaluzny, J, Hilditch, R.W., Clement, C. \& Rucinski, S.M. 1998, MNRAS, 296,347

Kovács, G., \& Jurcsik, J. 1996, ApJ, 466, L17

Kovács, G., \& Walker, A.R. 1998, ApJ, submitted

Kraft, R.P., Sneden, C., Langer, G. E. \& Prosser, C.F. 1992, AJ, 104, 645

Kraft, R.P., Sneden, C., Smith, G.H., Shetrone, M.D., Langer, G.E. \& Pilachowski, C.A. 1997, AJ, 113, 279

Lee, Y.-W. 1991 ApJ, 373, L43

Lee, Y. -W., Demarque, P., \& Zinn, R. J. 1990, ApJ, 350, 155

Lee, Y. -W., Demarque, P., \& Zinn, R. J. 1994, ApJ, 423, 248

McVean, J.R., Milone, E.F., Mateo, M. \& Yan, L. 1997, ApJ, 481, 782

Minniti, D., Geisler, D., Peterson, R.C. \& Claria, J.J. 1993, ApJ, 413, 548

Nissen, P., Gustafsson, B., Edvardsson, B. \& Gilmore, G. 1994, A\&A, 285, 440

Paczyński, B. 1997, in The Extragalactic Distance Scale, eds. M. Livio, M. Donahue \& N. Panagia (Cambridge Univ. Press, Cambridge) 273

Pont, F., Mayor, M, Turon, C. \& VanDenberg, D.A. 1998, A\&A, 329, 87

Rees, R.F. 1996, in Formation of the Galactic Halo .... Inside and Out, eds. H. Morrison \& A. Sarajedini (San Fransico: ASP), 289

Reid, I.N., 1996, MNRAS, 278, 367

Reid, I.N., 1997, AJ, 114, 161

Reid, I.N., 1998, AJ, 115, 204

Renzini, A. 1991, in Observational Tests of Cosmological Inflation, eds. T. Shanks, et al., (Dordrecht: Kluwer), 131

Renzini, A., Bragaglia, A., Ferraro, F.R., Gilmozzi, R., Ortolani, S., Holberg, J.B., Liebert, J., Wesemael, F. \& Bohlin, R.C. 1996, ApJ, 465, L23

Sandage, A. R. 1981a, ApJ, 244, L23 
Sandage, A. R. 1981b, ApJ, 248, 161

Simon, N.R., \& Clement, C.M. 1993, ApJ, 410, 526

Sneden, C., Kraft, R.P., Prosser, C.F. \& Langer, G. E. 1991, AJ, 102, 2001

Sneden, C., Kraft, R.P., Prosser, C.F. \& Langer, G. E. 1992, AJ, 104, 2121

Walker, A.R. 1992, ApJ, 390, L81

Walker, A.R. 1994, AJ, 108, 555

Zinn, R. 1985, ApJ, 293, 424

Zinn, R. \& West, M. 1984, ApJS, 55, 45 